\title{
Human hepatic stem cells transplanted into a fulminant hepatic failure Alb-TRECK/SCID mouse model exhibit liver reconstitution and drug metabolism capabilities
}

Ran-Ran Zhang ${ }^{1 \dagger}$, Yun-Wen Zheng ${ }^{1,2^{*}+}$, Bin $\mathrm{Li}^{3}$, Tomonori Tsuchida ${ }^{1}$, Yasuharu Ueno ${ }^{1}$, Yun-Zhong Nie ${ }^{1}$ and Hideki Taniguchi, ${ }^{1,4^{*}}$

\begin{abstract}
Introduction: Chimeric mice with humanized livers were recently established by transplanting human hepatocytes. This mouse model that is repopulated with functional human hepatocytes could be a useful tool for investigating human hepatic cell biology and drug metabolism and for other preclinical applications. Successfully transplanting human hepatocytes into mice requires that recipient mice with liver failure do not reject these human cells and provide a suitable microenvironment (supportive niche) to promote human donor cell expansion and differentiation. To overcome the limitations of current mouse models, we used Alb-TRECKISCID mice for in vivo human immature hepatocyte differentiation and humanized liver generation.

Methods: $1.5 \mu \mathrm{g} / \mathrm{kg}$ diphtheria toxin was administrated into 8-week-old Alb-TRECK/SCID mice, and the degree of liver damage was assessed by serum aspartate aminotransferase activity levels. Forty-eight hours later, mice livers were sampled for histological analyses, and the human donor cells were then transplanted into mice livers on the same day. Chimeric rate and survival rate after cell transplantation was evaluated. Expressions of human hepatic-related genes were detected. A human albumin enzyme-linked immunosorbent assay was performed after 50 days of transplantation. On day 60 after transplantation, drug metabolism was examined in mice.

Results: Both human primary fetal liver cells and hepatic stem cells were successfully repopulated in the livers of Alb-TRECK/SCID mice that developed lethal fulminant hepatic failure after administering diphtheria toxin; the repopulation rate in some mice was nearly $100 \%$. Compared with human primary fetal liver cells, human hepatic stem cell transplantation rescued Alb-TRECK/SCID mice with lethal fulminant hepatic failure, and human hepatic stem cell-derived humanized livers secreted more human albumin into mouse sera and also functioned as a "human liver" that could metabolize the drugs ketoprofen and debrisoquine.

Conclusion: Our model of a humanized liver in Alb-TRECK/SCID mice may provide for functional applications such as drug metabolism, drug to drug interactions, and promote other in vivo and in vitro studies.
\end{abstract}

\footnotetext{
* Correspondence: ywzheng@md.tsukuba.ac.jp; rtanigu@med.yokohama-cu.ac.jp

${ }^{\dagger}$ Equal contributors

'Department of Regenerative Medicine, Graduate School of Medicine,

Yokohama City University, 3-9 Fuku-ura, Kanazawa-ku, Yokohama, Kanagawa

236-0004, Japan

${ }^{4}$ Advanced Medical Research Center, Yokohama City University, 3-9 Fuku-ura,

Kanazawa-ku, Yokohama, Kanagawa 236-0004, Japan

Full list of author information is available at the end of the article
}

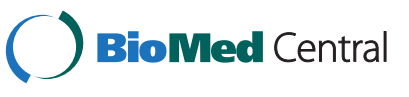

(C) 2015 Zhang et al.; licensee BioMed Central. This is an Open Access article distributed under the terms of the Creative Commons Attribution License (http://creativecommons.org/licenses/by/4.0), which permits unrestricted use, distribution, and reproduction in any medium, provided the original work is properly credited. The Creative Commons Public Domain Dedication waiver (http://creativecommons.org/publicdomain/zero/1.0/) applies to the data made available in this article, unless otherwise stated. 


\section{Introduction}

Because biomedical research cannot be performed in humans, investigators commonly use mice for pharmaceutical testing [1], although these models are not always useful. Most medically used drugs are primarily metabolized in the liver. However, the same drug can be metabolized into different metabolites in mouse and human livers due to species differences. Thus, it is quite often difficult to determine whether a potential drug poses any risks during development for clinical applications [2,3].

To address this problem, "humanized" mouse livers were developed by growing human liver tissues inside mice [4-6]. These models exhibited responses to drugs similar to those of the human liver. Current mouse models used for humanized liver generation are primarily $\mathrm{uPA}+/+$ (uroplasminogen activator) mice [4,7], Fah-/- (fumarylacetoacetate hydrolase) mice [6], and a recently reported TK-NOG (thymidine kinase) mouse.

However, previous reports showed that transplanted human immature cells or stem cells were less competitive as compared with human adult hepatocytes in Alb-uPAtg $(+/-) \operatorname{Rag} 2(-/-)$ mouse livers [8-10]. Moreover, Fah-/mice could only provide a growth advantage for differentiated hepatocytes but not for immature liver progenitor cells [11]. In our laboratory, we also failed to transplant human hepatic stem cells (HpSCs) into TK-NOG mice. Thus, no useful mouse model for the efficient engraftment of human immature liver cells currently exists.

To overcome this problem, we report here on a novel Alb-TRECK/SCID mouse model that could be efficiently repopulated with human immature hepatocytes. This transgenic mouse expresses human heparin-binding epidermal growth factor-like receptor (HB-EGF)-like receptors under the control of a liver cell-specific albumin promoter. After administering diphtheria toxin (DT), this model mouse developed fulminant hepatitis due to conditionally ablated hepatocytes, which provided space for donor cell residency and proliferation [12]. Previous studies successfully transplanted mouse hepatocytes into Alb-TRECK/SCID mice $[13,14]$, but there have been no reports of generating a humanized liver using Alb-TRECK/SCID mice.

In this study, we generated humanized livers in AlbTRECK/SCID mice by transplanting human primary fetal liver cells (FLCs) and HpSCs. This humanized liver provided an in vivo environment for universal stem cell differentiation and also an opportunity to predict the patterns of human drug metabolism and drug-to-drug interactions.

\section{Methods}

Acute liver injury mouse model

Alb-TRECK/SCID mice were provided by our collaborators at the Tokyo Metropolitan Institute of Medical Science. Homozygosity was confirmed by backcrossing for at least three generations. Alb-TRECK/SCID mice were housed at Yokohama City University. Animal experimental work was conducted in accordance with the Guidelines for Proper Conduct of Animal Experiments (Science Council of Japan), and all experimental procedures were approved by the institutional review board of the Animal Research Center, Yokohama City University School of Medicine (No.075).

DT (Sigma, St Louis, MO, USA; D0564-1MG) was intraperitoneally administered $(1.5 \mu \mathrm{g} / \mathrm{kg})$ to 8 -week-old Alb-TRECK/SCID mice, and the degree of liver damage was assessed by serum aspartate aminotransferase (AST) activity levels.

\section{Donor cell culture}

Human primary FLCs of embryonic age between weeks 14 and 18 were obtained from Cell Systems (Kirkland, WA, USA). This study was conducted with the approval of the ethics committee of Yokohama City University (Approval No. A100903011).

Human primary FLCs were cultured in Dulbecco's modified Eagle's medium with Ham's F-12 nutrient mixture (1:1 mixture; Sigma, St. Louis, MO, USA) supplemented with $10 \%$ fetal bovine serum, human $\gamma$-insulin $(1.0 \mu \mathrm{g} / \mathrm{mL}$; Wako, Tokyo, Japan), nicotinamide (10 mM; Sigma), dexamethasone (100 nM; Sigma), and Lglutamine ( $2 \mathrm{mM}$; Gibco, Carlsbad, CA, USA) in dishes coated with type IV collagen (Becton Dickinson Labware). After the first 24 hours of culture, human recombinant hepatocyte growth factor (50 ng/mL; Sigma) and epidermal growth factor $(10 \mathrm{ng} / \mathrm{mL}$; Sigma) were added.

For cell passaging, culture medium was removed, cells were treated with $0.05 \%$ trypsin- ethylenediaminetetraacetic acid (Gibco) at room temperature for 5 minutes and then gently detached from the dish. Suspended cells were neutralized and washed with culture medium that contained 10\% fetal bovine serum. The viability of dissociated cells was never $<90 \%$ based on trypan blue exclusion.

Human HpSCs were isolated using a DakoCytomation MoFlo high-speed cell sorter (Beckman Coulter, Pasadena, CA, USA) with cell antigens that included CDCP1, CD90, and $\mathrm{CD} 66$ for a $\mathrm{CDCP} 1^{+} \mathrm{CD} 90^{+} \mathrm{CD} 66^{-}$population. Details for cell isolation were previously described [15]. These cells were cultured using the same procedures as for human primary FLCs.

\section{Liver biochemistry tests}

After DT injection, blood samples were obtained from a mouse tail vein every 24 hour and centrifuged at 4,000 rpm at $4^{\circ} \mathrm{C}$ for 20 minutes. Serum samples were assayed for serum AST activity measured with a FUJIFILM Kit according to the manufacturer's instructions (FUJIFILM, Tokyo, Japan). Serum AST activity levels of mice used for cell transplantation was measured at 48 hours after DT injection. 


\section{Cell transplantation}

Prior to cell transplantation, serum AST activity levels were checked, and mice with AST values between 12,000 and 16,000 IU/L were used as recipients. When cultured human primary FLCs or HpSCs reached 90\% confluence, these cells were detached and adjusted to a final concentration of $1 \times 10^{6}$ viable cells per $50 \mu \mathrm{L}$ culture medium. Human primary FLCs or HpSCs $\left(1 \times 10^{6}\right)$ were transplanted into the spleens of Alb-TRECK/SCID mice. Mice in the sham group received $50 \mu \mathrm{L}$ sterile saline.

\section{BrdU injection}

At 46 hours after DT was injected into Alb-TRECK/ SCID mice, BrdU $(50 \mathrm{mg} / \mathrm{kg})$ was administered intraperitoneally to five mice in each group, and mice were sacrificed 2 hours later. Liver sections were prepared, fixed with $4 \%$ paraformaldehyde (PFA) and washed with 0.05\% Tween 20 in phosphate-buffered saline (PBS). Sections were then treated with $2 \mathrm{~N}$ hydrochloric acid and neutralized with $0.1 \mathrm{M}$ sodium tetraborate ( $\mathrm{pH}$ 8.5). The sections were then stained with an anti-BrdU antibody (BD Pharmingen, San Jose, CA, USA), and Alexa Fluor 488 goat anti-mouse IgG1 (Invitrogen, Carlsbad, CA, USA) was used as a secondary antibody for visualization. Nuclei were stained with 4',6-diamidino-2-phenylindole (DAPI), and sections were mounted with Apathy's Mounting Media (Wako Pure Chemical Industries, Osaka, Japan).

\section{Histology and immunocytochemistry}

Liver tissues were fixed with $10 \%$ neutral formalin for 2 days and washed with PBS for 1 day. After dehydration with ethanol and xylene, tissues were embedded in paraffin and serial sections were prepared ( $4 \mu \mathrm{m}$ thick). These samples were stained with hematoxylin and eosin.

For double or triple immunohistochemical staining, liver tissues were frozen in optimum cutting temperature compound (Sakura, Tokyo, Japan), liver sections (5 um thick) were prepared and fixed in acetone:methanol (1:1) for 30 minutes, and then blocked with 10\% normal goat serum for 60 minutes. Sections were then incubated with primary antibodies (1:200), including mouse anti-human albumin mAb (Sigma), mouse anti-human CK19 mAb (Dako, Tokyo, Japan), guinea pig anti-human CK8/18 (Progen, Heidelberg, Germany), mouse anti-human nuclei (Millipore, Billerica, MA, USA), and mouse anti-Ki67 (Dako) at $4^{\circ} \mathrm{C}$ overnight. Sections were washed with PBS and then incubated with appropriate Alexa-488, -555, or -647-conjugated secondary antibodies (1:500; Invitrogen) at room temperature for 60 minutes. Cells were counterstained with DAPI and sections were mounted with Apathy's Mounting Media (Wako Pure Chemical Industries). Images were acquired using a Zeiss AxioImager and microscope (Carl Zeiss, Jena, Germany).

\section{Real-time PCR}

Total RNAs from humanized liver tissue and human primary FLCs and HpSCs and human adult hepatocytes were extracted using Isogen reagent (Nippon Gene, Toyama, Japan). cDNA was synthesized with a High Capacity cDNA Reverse Transcription Kit (Applied Biosystems, Foster, CA, USA). Quantitative PCR was performed according to the manufacturer's protocol using TaqMan Gene Expression Assays (Applied Biosystems) and data were analyzed with an ABI PRISM ${ }^{\circ} 7900 \mathrm{HT}$ Sequence Detection System (Applied Biosystems). Probes used were ALB (Hs00609411_m1), AFP (Hs01040607_m1), CYP3A4 (Hs01546612_m1), CYP2C9 (Hs00426397_m1), CYP2C19 (Hs00426380_m1), and hACTB (4326315E). TaqMan Gene Expression Assay IDs (Applied Biosystems) are shown in parentheses after the gene names.

\section{Microarray analysis}

Total RNAs were extracted from human primary FLCs, HpSCs, and Alb-TRECK/SCID mouse livers that received cell transplants separately for three independent experiments using an RNeasy Mini Kit (Qiagen, Venlo, Netherlands). RNA samples were individually hybridized to a pool of two commercial normal ovary RNA on a Whole Human Genome Agilent $4 \times 44 \mathrm{~K}$ v2 Oligonucleotide Microarray (Agilent Technologies, Santa Clara, CA, USA), according to the manufacturer's instructions. For crossspecies comparisons of expression profiles, total expression data at the gene level were cross-referenced to other species using the HomoloGene IDs in the Mouse Genome Informatics curated data set of human-mouse orthology with Phenotype Annotations [16]. To generate a heat map, we used a hierarchical clustering method with Euclidean distances for complete linkage on GeneSpring11.5.1. to analyze 83 and 38 selected gene expression profiles. The raw data of the microarray analysis have been deposited in the Gene Expression Omnibus database (GSE62933).

\section{Albumin assay}

Blood samples $(20 \mu \mathrm{l})$ were collected periodically from mouse tail veins and centrifuged at $4,000 \mathrm{rpm}$ at $4^{\circ} \mathrm{C}$ for 20 minutes. Serum samples were assayed for human albumin using a human albumin enzyme-linked immunosorbent assay (ELISA) quantitation kit (Bethyl Laboratories Inc., Montgomery, TX, USA), according to the manufacturer's instructions. After 6 minutes, reactions were stopped by adding $2 \mathrm{M}$ sulfuric acid, and absorbance was read at $450 \mathrm{~nm}$ using a Multimode Detector DTX 880 (Beckman Coulter, Pasadena, CA, USA).

\section{Drug metabolite detection}

At about 7 to 8 weeks, mice without and with cell transplantation were intravenously administrated ketoprofen $(15 \mathrm{mg} / \mathrm{kg})$, and urine samples were collected from 0 to 
2 hours in $0.5 \mathrm{M}$ acetate buffer ( $\mathrm{pH}$ 5.0). For debrisoquine (DEB) metabolic testing, mice were administered DEB (2 mgkg) by oral gavage, and then blood samples were obtained from tail veins at $0,0.5,1,2,4$, and 8 hours with heparin-Na added. Plasma was separated from blood by centrifugation. Metabolites were quantified using an LC-20A Series liquid chromatographytandem mass spectrometer (Shimadzu, Kyoto, Japan) with an Intersil ODS-3 column (GL Science Co., Tokyo, Japan). The details were previously described [17].

\section{Statistical analysis}

Results for two groups were statistically compared using the Mann-Whitney U-test and results for more than two groups were compared by one-way analysis of variance and Bonferroni multiple comparisons tests. A logrank (Mantel-Cox) test and the Kaplan-Meier method were used to assess post-transplantation survival. A $P$ value of $<0.05$ was considered significant. Statistical analysis was performed using Graphpad Prism software (San Diego, CA, USA).

\section{Results}

Diphtheria toxin induces lethal fulminant hepatic failure in Alb-TRECK/SCID mice

Alb-TRECK/SCID mice hepatocytes harbor the gene for the human DT receptor, HB-EGF, under the control of an albumin promoter, and exhibit cytotoxic effects after DT administration. To evaluate the effects of DT injection on liver injury, we injected DT doses of $0.5,1,1.5$, 2 , and $5 \mu \mathrm{g} / \mathrm{kg}$ into groups of 8-week-old Alb-TRECK/ SCID mice (five per group) and measured serum AST activity levels every 24 hours up to 96 hours after administration of DT.

Serum AST activity reached a peak at 48 hours after DT injection and then returned toward basal levels by 96 hours (Figure 1A). This indicated that acute liver failure might have occurred and that the most severe liver damage might have been induced 48 hours after the administration of DT. All mice were dead within 48 hours after receiving 2 and $5 \mu \mathrm{g} / \mathrm{kg}$ of DT, and three mice were dead by 72 hours while two mice survived after administration of $1.5 \mu \mathrm{g} / \mathrm{kg}$ DT. All mice survived after administration of 0.5 and $1 \mu \mathrm{g} / \mathrm{kg}$ DT. Thus, we defined $1.5 \mu \mathrm{g} / \mathrm{kg}$ DT for 48 hours as a "sub-lethal dose" that could induce fulminant hepatic failure.

Next, we histologically assessed pathological changes in the liver at 48 hours after administration of DT in 8week-old mice with different serum AST activities. This showed that, as compared with normal mice which were not administered DT, after administration of DT there was a disorganized hepatic architecture showing a correction for congestion with the increased serum AST activity, and hepatocytes also had multiple, deeply stained acidophilic cytoplasmic inclusions along with dark nuclei (most probably apoptotic hepatocytes), while other hepatocytes appeared either preserved or exhibited a vacuolated cytoplasm with dark nuclei, along with little or no portal vein inflammation (Figure 1B and Additional file 1: Figure S1).

Furthermore, after DT injection, all of the mice with AST values of $<8,000 \mathrm{IU} / \mathrm{L}$ had survived, the survival rate of mice with AST values between 8,000 and $12,000 \mathrm{IU} / \mathrm{L}$ declined to about $60 \%$, and it further declined to $25 \%$ when the AST values were between 12,000 and 16,000 IU/L. All of the mice with serum AST activity levels of $>16,000 \mathrm{IU} / \mathrm{L}$ were dead within 48 hours (Figure 1C). These results were in agreement with those of a previous study that showed that Alb-TRECK/SCID mice were an ideal lethal fulminant hepatic failure model generated by only a single DT injection $[12,14]$.

\section{Mouse hepatocyte proliferation is induced in response to fulminant hepatic liver failure}

To assess in vivo hepatocyte proliferation after the administration of DT, we performed an immunohistochemical analysis using cell cycle markers for total cell cycle activity (Ki-67) and S-phase progression (BrdU incorporation, Cyclin A) in the livers of both normal mice and mice with fulminant hepatic liver failure. This showed that there was a higher degree of Ki67-positive expression (Figure 2A, lower panels) and BrdU incorporation (Figure 2B, lower panels) in livers at 48 hours after the administration of DT. In contrast, no positive Ki67 (Figure 2A, upper panels) and only a few BrdU-positive cells (Figure 2B, upper panels) were detected in normal mouse livers. These results showed that mouse liver regeneration was occurring after DT injection.

\section{Alb-TRECK/SCID mice with lethal fulminant hepatic failure} are rescued by human hepatic stem cell transplantation

Human immature hepatocytes, including human primary FLCs and HpSCs, could be used for long-term in vitro culture. Human $\mathrm{HpSC}$ exhibited uniform cell morphology, with more ALB and fewer CK19-positive cells as compared with human primary FLCs (Additional file 1: Figure S2). Eight-week-old mice received DT injections $(1.5 \mu \mathrm{g} / \mathrm{kg}) 2$ days before cell transplantation and were checked for serum AST activity. Mice with AST activity levels between 12,000 and 16,000 IU/L were used as recipients and were transplanted with $1 \times 10^{6}$ cells, either human primary FLCs or HpSCs as described in the experimental protocol (Figure 3A).

More than $60 \%$ of human HpSCs transplanted mice survived for more than 120 days, whereas all of the human primary FLCs transplanted mice were dead within 110 days (Figure 3B). However, human primary FLCs rescued mice in terms of survival at 7 days after 


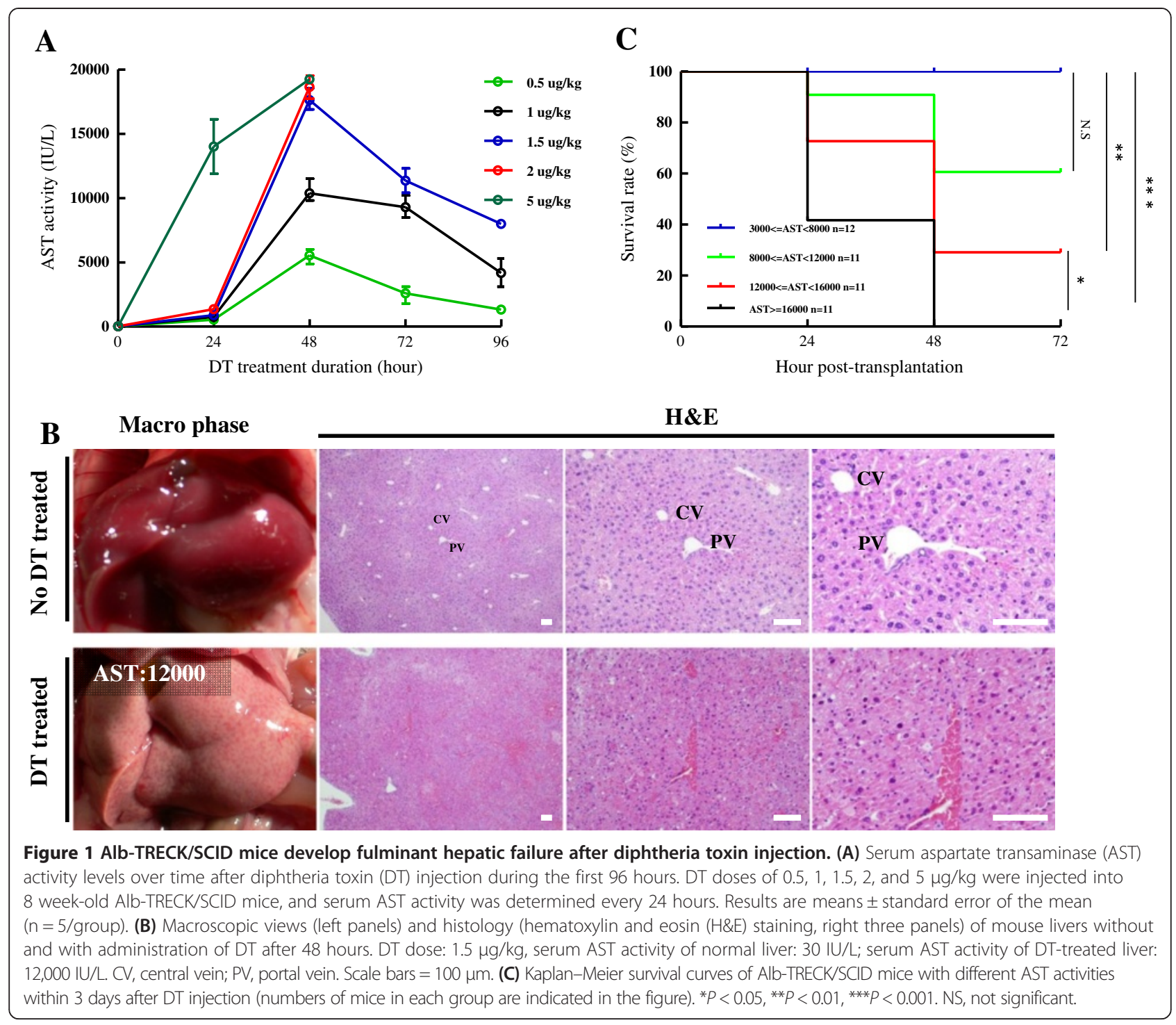

transplantation, as $>80 \%$ of the sham group mice with saline transplantation were dead within 7 days. Also, mice with human primary FLCs exhibited a pronounced body weight loss within 3 days after cell transplantation, whereas mice that received human $\mathrm{HpSC}$ s showed a gradual body weight increase similar to that of the sham group (Additional file 1: Figure S3).

Whole mouse livers at 6 weeks after cell transplantation were grossly examined under a microscope (Figure 3C, left panels) and by histological examination, which showed that both human primary FLCs and HpSCs had reconstituted the liver structure by replacing original mouse hepatocytes (Figure 3C, middle and right panels). Macroscopically, at 4 days after human $\mathrm{HpSC}$ transplantation, we detected small human hepatic clusters that were uniformly distributed around the liver and had proliferated into large clusters at 45 days (Additional file 1: Figure S4A). Thus, these whole mouse livers that had been replaced with human hepatocytes were designated "humanized livers" (Additional file 1: Figure S4B). These results showed that mice with lethal fulminant hepatic failure that underwent human primary FLC transplantation survived over a short term, whereas for long-term survival transplanted human $\mathrm{HpSC}$ might have been functional. Both human primary FLC- and HpSC-derived humanized livers were healthy and exhibited liver structures similar to normal mouse livers.

\section{Proliferative human hepatic stem cells successfully} differentiate in humanized livers of Alb-TRECK/SCID mice Prior to testing whether the transplanted human immature hepatocytes in mice had the potential for differentiation and be functional in vivo, we firstly confirmed human hepatocytes existed in mouse livers by immunohistochemical analysis at about 6 weeks after transplanting human primary FLCs (Figure 4A, left panels) or HpSCs (Figure 4B, 

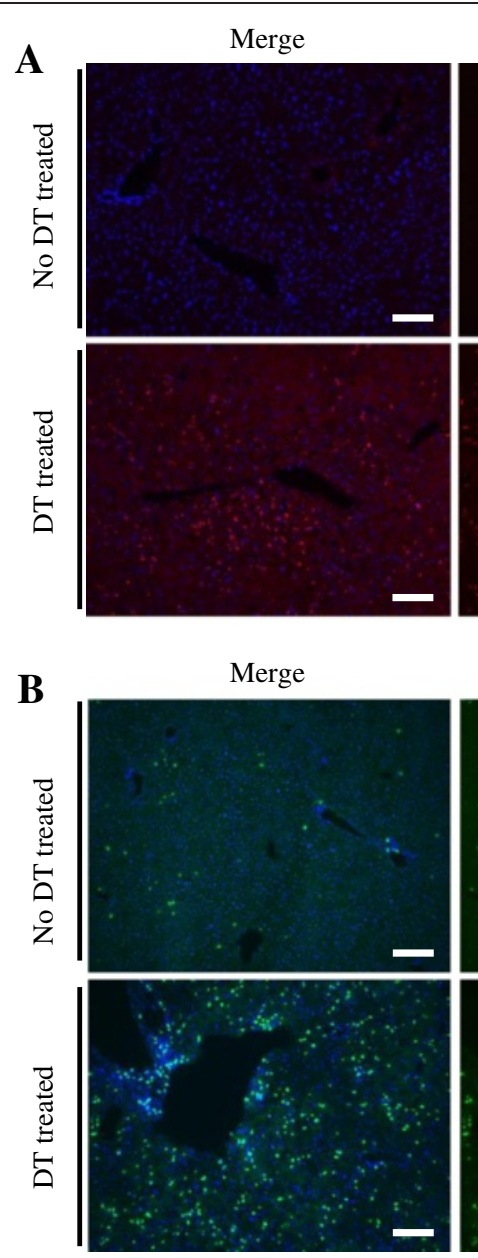
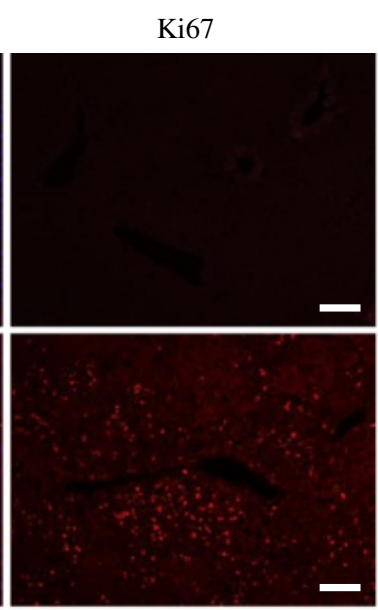

BrdU
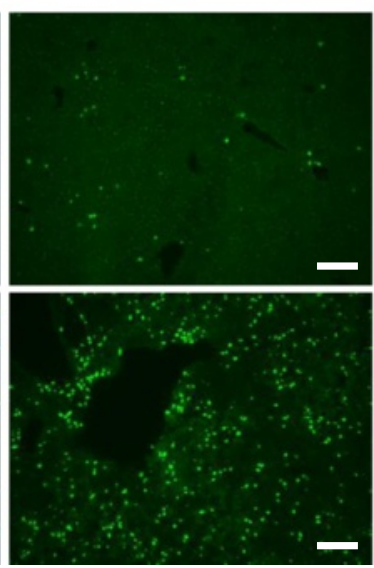

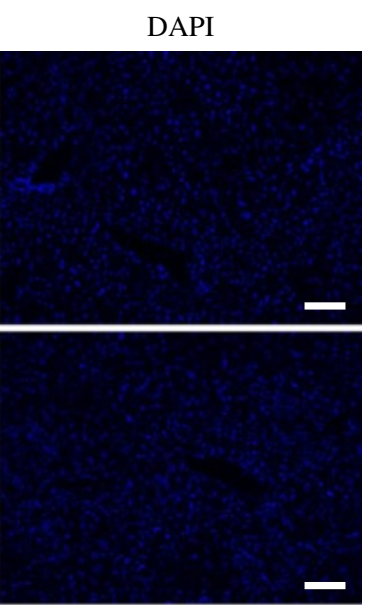

DAPI

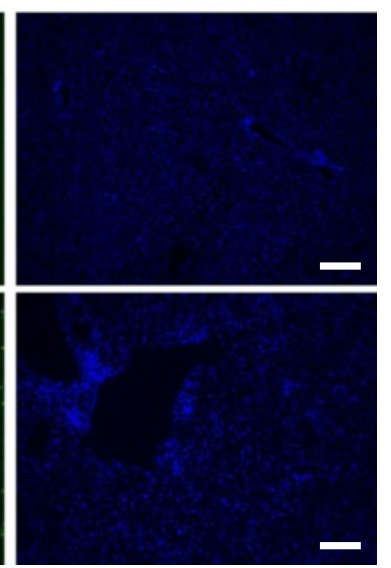

Figure 2 Extensive mouse hepatocyte proliferation after administration of diphtheria toxin. Immunofluorescent staining for cell proliferation markers Ki67 (A) and BrdU (B) in liver tissues from 8-week-old Alb-TRECK/SCID mice without and with diphtheria toxin (DT) treatment. DT-treated mouse liver with serum aspartate aminotransferase activity of $12,000 \mathrm{IU} / \mathrm{L}$ was sampled at 48 hours. At 2 hours before sampling, BrdU $(50 \mathrm{mg} / \mathrm{kg}$ ) was administered intraperitoneally. Experiments were performed with five mice/group, and representative images are shown. Nuclei were counterstained with 4',6-diamidino-2-phenylindole (DAPI). Scale bars $=100 \mu \mathrm{m}$.

left panels). Liver sections from these mice that were specifically positively co-stained with human nuclei and human cytokeratin 8/18 (CK8/18) antibodies were donor cellderived human hepatocytes, while the original liver regions in mice were negative for these markers.

To assess the degree of cell differentiation in vivo, we immunohistochemically assessed for human albumin (ALB) and human cytokeratin 19 (CK19) expressions. This showed that human primary FLC-derived liver sections that were positively stained with human CK8/18 were human ALB and CK19 negative (Figure $4 \mathrm{~A}$, right panels), whereas human $\mathrm{HpSC}$-derived human hepatocytes in mouse livers were well differentiated and with upregulated human ALB expression. Human ALB-positive hepatocytes that were CK19 negative resembled functional hepatocytes, while cells that positively co-stained with human ALB and CK19 exhibited a bipotential capability with differentiation into hepatocytes and cholangiocytes (Figure 4B, right panels).

A large-scale scan method to analyze the entirety of human HpSC-derived humanized liver lobes showed that multiple round and colony-like clusters were distributed around the liver lobes with clear human nuclei, and CK8/ 18 and ALB expression (Additional file 1: Figure S5), indicating that the colony-forming capability of human HpSCs were maintained in humanized livers.

These results showed that, compared with human primary FLCs, human HpSCs had a high potential for differentiation into functional hepatocytes in vivo in response to the rescue of damaged mouse liver functions.

\section{Characterization of human drug metabolism gene} expression in Alb-TRECK/SCID mouse with humanized livers We also evaluated human drug metabolism-related gene expression by quantitative PCR and microarray analysis 


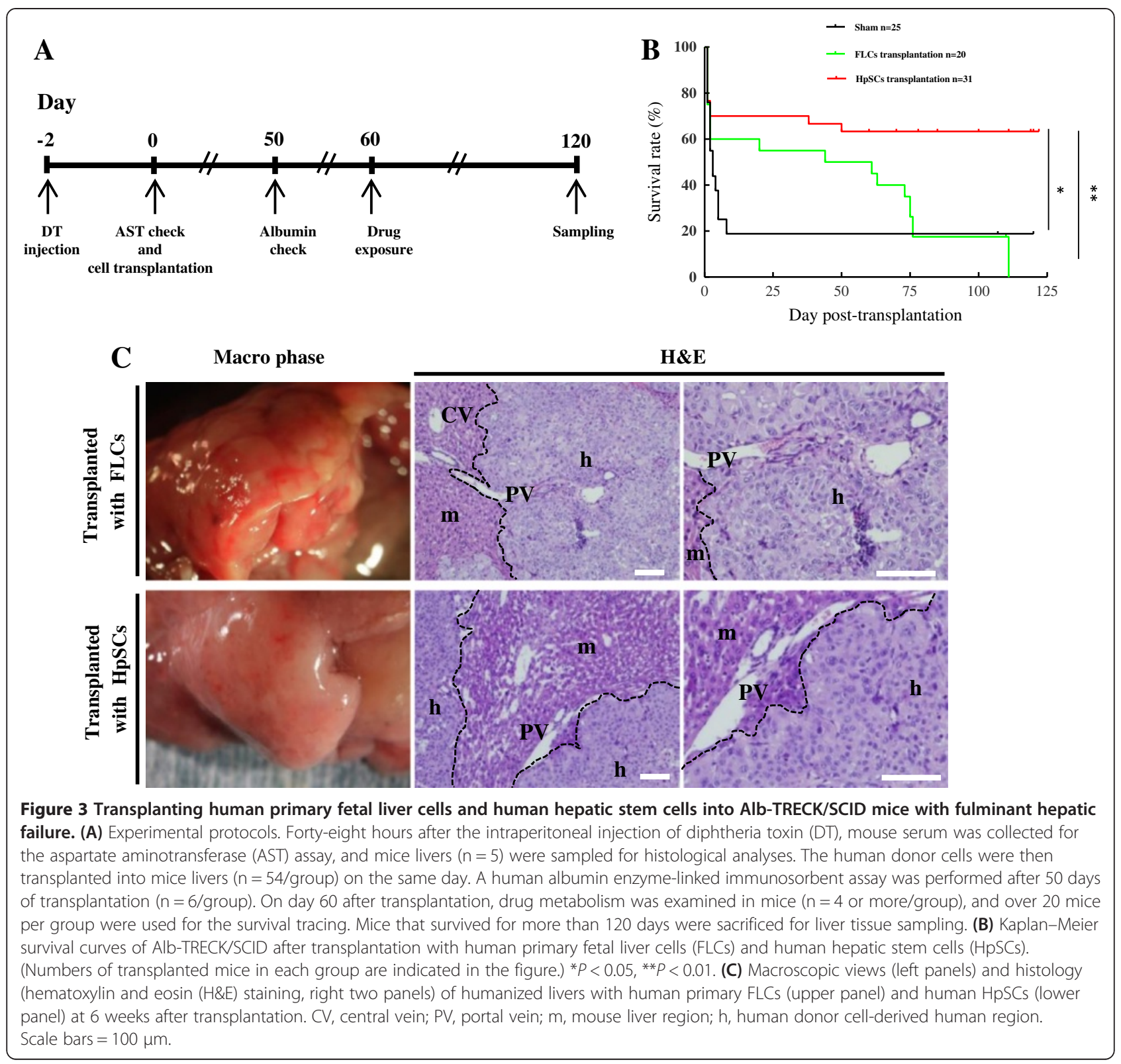

to assess whether human primary FLC- and HpSCderived humanized livers could be used for human drug metabolism studies. At 8 weeks after transplantation, we observed very high gene expression levels associated with the human hepatic functional markers ALB, AFP, and cytochrome P450, including CYP3A4, 2C9, and $2 \mathrm{C} 19$, which collectively metabolize over $80 \%$ of clinical drugs (Figure 5A). Almost none of the probes on the human gene expression array had cross-hybridized with murine mRNA.

To comprehensively assess for genes associated with drug metabolizing enzymes, we performed microarray analysis for 83 previously reported human drug metabolismrelated genes (Figure 5B) and 38 mature hepatocyte- specific genes (Additional file 1: Figure S6A) whose expressions were robustly increased in humanized livers. We chose the 83 genes because their expression increased continuously during both murine and human liver development [18], and the subset of 38 genes was used to identify differentiated hepatic characteristics [19]. Three pairwise comparisons selectively displayed genes with a twofold expression change (increase or decrease) in humanized livers derived from human HpSCs, human HpSCs and human adult hepatocytes, and showed that humanized livers shared 1,049 genes with human adult hepatocytes, which included liver-specific genes, ALB, $\mathrm{AFP}$ and $\mathrm{ABCC6}$, and genes for drug metabolizing enzymes, CYP2C9, 2C19 and 2D6 (Additional file 1: Figure 

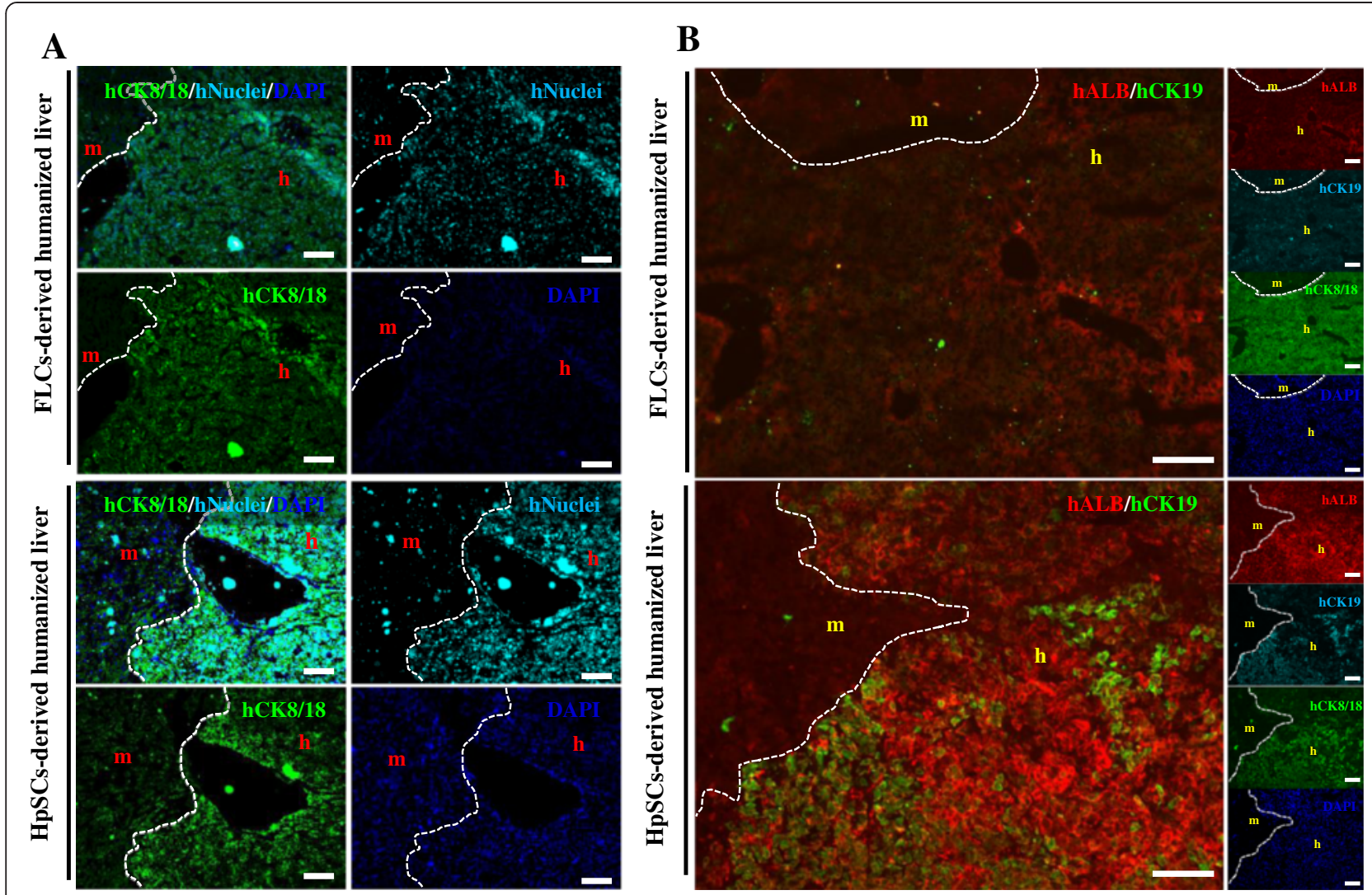

Figure 4 Characterization of human primary fetal liver cell- and human hepatic stem cell-derived human hepatocytes in Alb-TRECK/ SCID mice. (A) Immunohistochemistry to distinguish between human hepatocytes stained with anti-human CK8/18 (green) antigen and anti-human nuclear antigen (aqua blue) in human primary fetal liver cell- (FLC; upper panels) and human hepatic stem cell- (HpSC; lower panels) derived humanized livers at 6 weeks after transplantation. (B) Immunohistochemistry analyses for human albumin, human CK19, and human CK8/ 18 expression in human primary FLC- (upper panels) and HpSC-(lower panels) derived livers at 6 weeks after transplantation. White dashed line: mouse liver region distinguished from human liver region. Nuclei were counterstained with 4',6-diamidino-2-phenylindole (DAPI, blue). Scale bars $=100 \mu \mathrm{m}$. $\mathrm{m}$, mouse liver region; $\mathrm{h}$, human donor cell-derived human region.

S6B and Additional file 2). We also found that 27 of 53 phase I, 86 of 99 phase II, and 35 of 51 phase III genes could be detected in humanized livers derived from human HpSCs, similar to that of human adult hepatocytes (Additional file 1: Figure S6C and Additional file 3). These results indicated that relevant functional human drug metabolizing enzymes were expressed in humanized livers derived from human HpSCs, which could be useful for preclinical drug development.

\section{Functional characterization of humanized livers in Alb-TRECK/SCID mice}

At about 8 weeks after transplantation, the level of liver repopulation with human donor cells and human ALB concentrations in mouse sera were determined. The average liver repopulation rate for human primary FLCand $\mathrm{HpSC}$-derived humanized livers were $76 \%$ and $71 \%$, respectively; no significant difference was observed. Several humanized livers reached liver repopulation levels of about $100 \%$, which indicated that almost the entire mouse liver had been reconstituted with human hepatocytes (Figure 6A). Humanized livers derived from human HpSCs resulted in more human ALB secretion than those from human primary FLCs, and no human ALB could be detected in mice after saline transplantation (Figure 6B). The drug metabolism profiles based on gene expression and human ALB secretion patterns suggested the potential of humanized livers derived from human HpSCs for early identification of major drug metabolites in vivo.

We administered ketoprofen (KTP) [20] and DEB [21], which are known to be metabolized differently by mice and humans, into sham-treated mice and mice transplanted with human hepatocytes. KTP, a CYP2C9 substrate, is primarily metabolized to KTP-glucuronide (KTP-glu) by humans and metabolized to hydroxyl-KTP in mice. DEB is a prototypical CYP2D6 substrate that is converted to its $4 \mathrm{OH}$ metabolite $(4 \mathrm{OH}$ DEB) by CYP2D6. The fold-change of KTP-glu/M1 was significantly greater in human $\mathrm{HpSC}$-derived humanized livers 


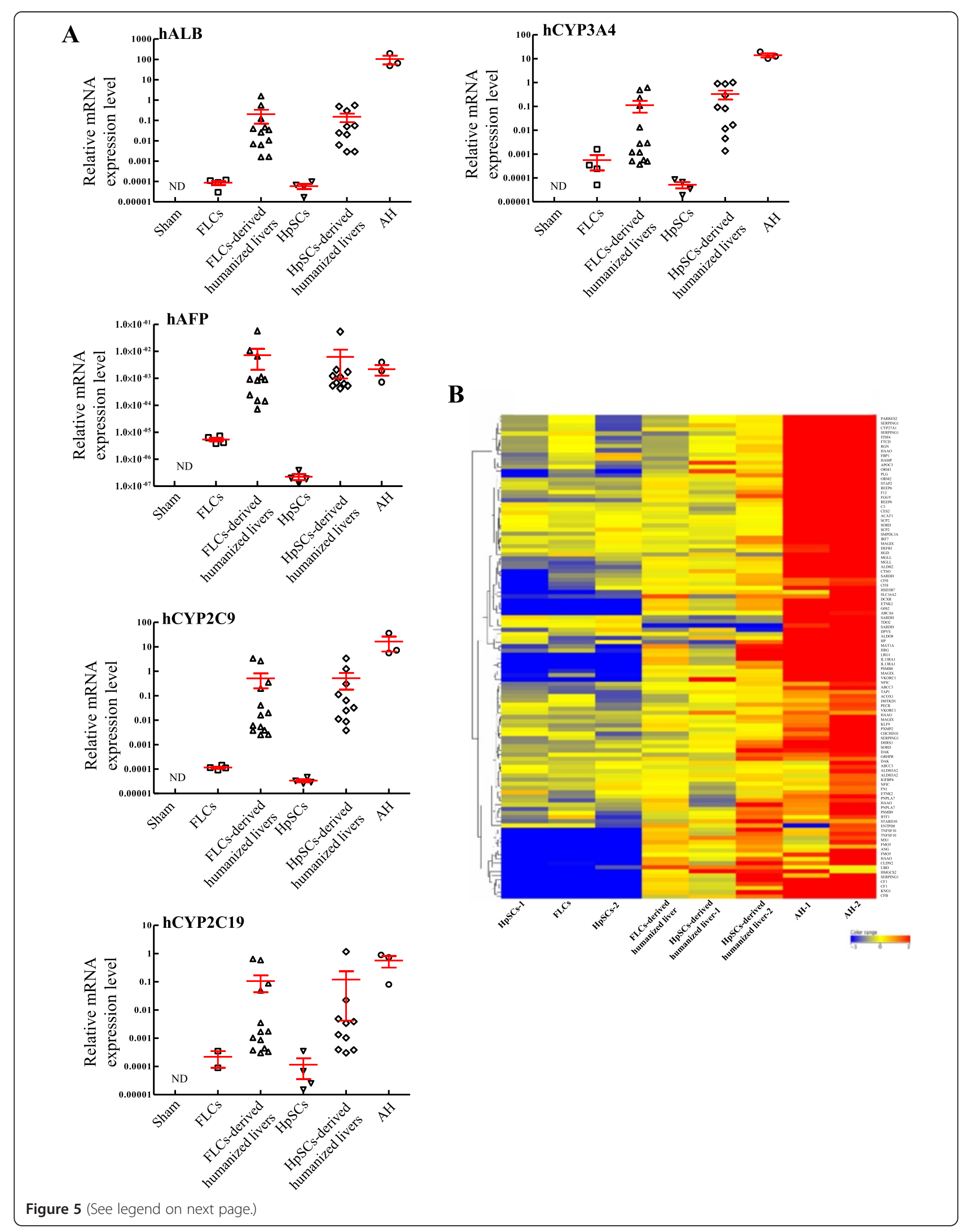


(See figure on previous page.)

Figure 5 Expressions of human hepatocyte-related genes in human primary fetal liver cell- and human hepatic stem cell-derived humanized livers. (A) Results of quantitative PCR for the expression of functional hepatocyte markers, including hALB, hAFP, hCYP3A4, hCYP2C9, and hCYP2C19, in samples taken before and about 8 weeks after human primary fetal liver cell (FLC) and human hepatic stem cell (HpSC) transplantation. Results are mean \pm standard error of the mean ( $n \geq 4$ mice/group). (B) Heat maps for 83 human-specific drug metabolism genes and transcription factors, shown separately for independent experiments to analyze human primary FLCs and HpSCs before and at 8 weeks after cell transplantation. Human adult hepatocytes were used as a positive control. AH, adult hepatocyte; ND, not detectable; Sham, mice transplanted with saline.

than in human primary FLC-derived humanized livers and sham-treated mice (Figure 6C). For DEB, a higher metabolic ratio of $4 \mathrm{OH}-\mathrm{DEB} / \mathrm{DEB}$ was detected in human HpSC-derived humanized livers (Figure 6D). These results showed that human HpSC-derived humanized livers would be advantageous for improving the quality of human drug metabolism and for preclinical studies.

\section{Discussion}

Recent studies have shown that mouse livers could be repopulated with human hepatocytes, including adult

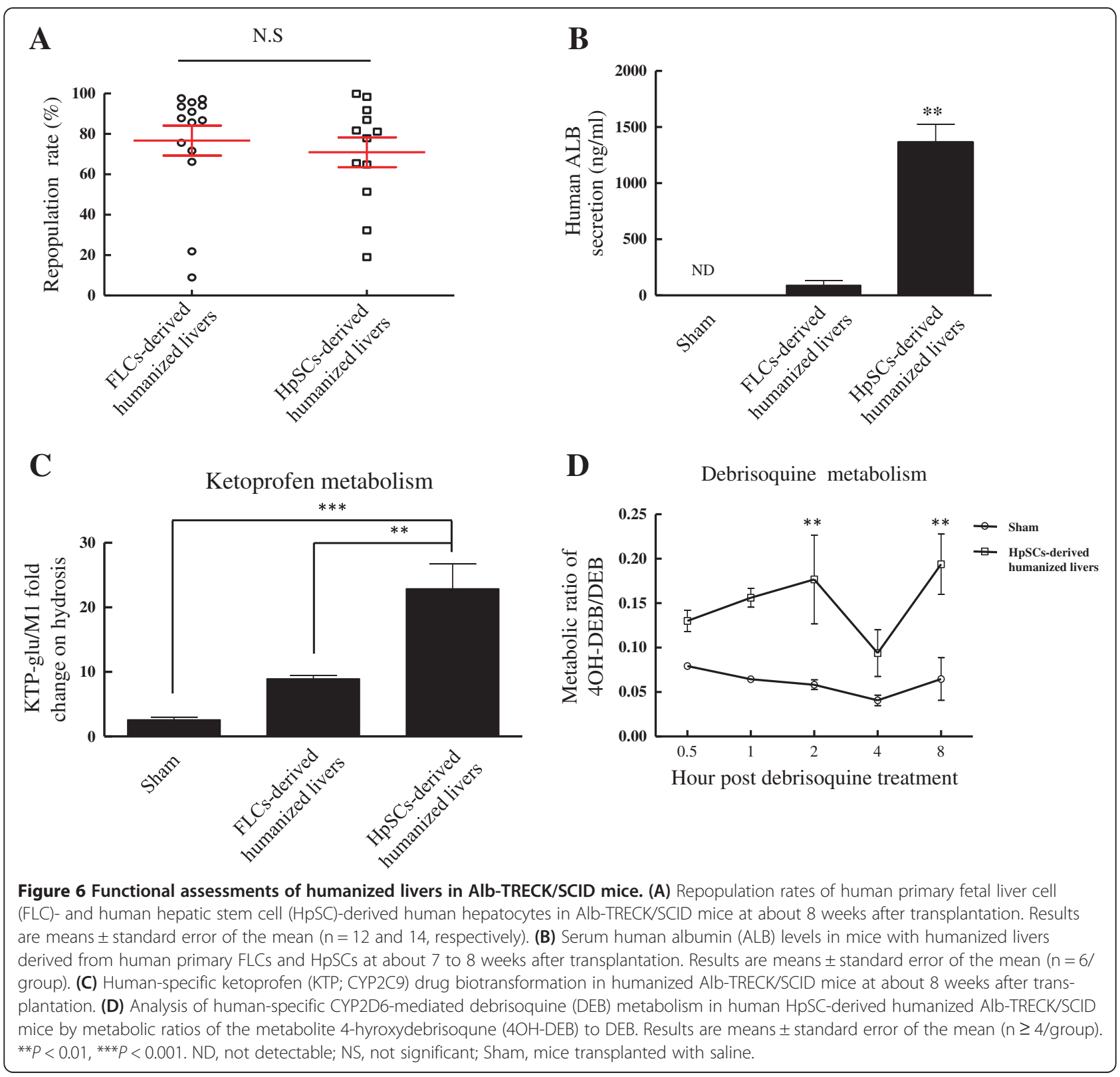


hepatocytes and proliferative $\mathrm{HpSCs}$ [22]. These were used as a preclinical experimental model for drug metabolism testing [23] and drug discovery and development [24], and they also provided an in vivo environment for cell maturation and differentiation [25]. The major aim of the present study was to generate a novel acute liver disease mouse model that provided for human immature hepatocyte proliferation, maturation, and differentiation in order to acquire drug metabolism activities.

It was clear that this novel Alb-TRECK/SCID mouse model developed lethal fulminant hepatic failure using one dose of DT, which provided a platform for studying the basic biology of liver regeneration after injury and liver disease development. As is known, even human immature hepatocytes can proliferate extensively in vitro, although they lose drug metabolism functions which limits their preclinical applications [26]. After transplanting human immature hepatocytes, including human primary FLCs and human HpSCs, into Alb-TRECK/ SCID mice with lethal fulminant hepatic failure, we found histological and immunohistochemical evidence that both human primary FLCs and HpSCs could expand and reconstitute the damaged mouse liver structures; the repopulation rate in some mice was nearly $100 \%$.

However, as compared with human primary FLCs, human $\mathrm{HpSC}$ transplantation promoted mouse survival and resulted in more human ALB secreted into mouse sera. These cells also exhibited maturation and differentiation in vivo with human drug metabolism activities, which indicated that humanized livers in mice derived from human $\mathrm{HpSC}$ were similar to a mature, functional "human organ" and have potential applications in drug development. This further confirmed that Alb-TRECK/ SCID mice were an ideal model for humanized liver generation.

Despite the prospects and advantages of Alb-TRECK/ SCID mice as an ideal model for human HpSC-derived humanized liver generation, there was one unique disadvantage as well. We transplanted human adult hepatocyte into three Alb-TRECK/SCID mice with one DT dose treatment and failed to get the humanized livers, possibly because human adult hepatocytes lacked the capability to proliferate. Additional DT doses cannot be administered after human adult hepatocyte transplantation. Since the DT receptor (HB-EGF) in Alb-TRECK/ SCID mice hepatocytes is under the control of the albumin promoter, additional DT treatment will destroy transplanted human adult hepatocytes in the mouse liver.

Thus, Alb-TRECK/SCID mice can sustain acute liver injuries with only one DT dose injection and are easily bred recipients for human immature hepatocyte transplantation. They are an acceptable model for human
HpSC transplantation as they provide a beneficial environment that allows for the differentiation into mature hepatocytes. These mice are certainly an interesting model that has tremendous potential applications, not only for ex vivo expansion of human hepatocytes, but also to test candidate therapeutic drugs for liver toxicity and metabolism as well as for drug screens.

\section{Conclusions}

Overall, we have shown that Alb-TRECK/SCID mice are an ideal model for induced lethal fulminant hepatic failure that could be used to study hepatocyte regeneration and liver disease development and facilitate in vivo human immature hepatocyte differentiation; it also has the potential for human drug metabolism testing. After human immature hepatocytes, including human primary FLCs and HpSCs, were transplanted into Alb-TRECK/ SCID mice administered DT, damaged mouse livers were reconstituted with high liver repopulation rates. Furthermore, human HpSC transplantation-derived humanized livers exhibited higher human liver functions, including human ALB secretion and drug metabolism capabilities. Thus, our model of humanized livers in Alb-TRECK/ SCID mice allows for the use of functional applications, such as examinations of drug metabolism, drug-drug interactions, and hepatic virus transfection, and the promotion of human $\mathrm{HpSC}$-related studies, such as in vivo evaluation of stem cell differentiation and development processes.

\section{Additional files}

\section{Additional file 1: Supplemental information. This file contains} Figure S1-S6.

Additional file 2: This file contains a list of global gene expression profiles in each of the comparisons, referred to as Figure S6B.

Additional file 3: This file contains a list of drug metabolism related phase I, phase II and phase III genes detected in human HpSCs, human HpSC-derived humanized liver and human adult hepatocytes, referred to as Figure S6C.

\section{Abbreviations}

ALB: albumin; AST: aspartate aminotransferase; BrdU: 5-bromo-2'deoxyuridine; CK19: cytokeratin 19; DAPI: 4',6-diamidino-2-phenylindole; DEB: debrisoquine; DMEM: Dulbecco's modified Eagle's medium; DT: diphtheria toxin; ELISA: enzyme-linked immunosorbent assay; FLC: fetal liver cell; HB-EGF: heparin-binding epidermal growth factor; HpSC: hepatic stem cell; KTP: ketoprofen; KTP-glu: KTP-glucuronide; PBS: phosphate-buffered saline; SCID: severe combined immunodeficiency; TRECK: toxin receptor mediated cell knockout.

\section{Competing interests}

The authors declare that they have no competing interests.

\section{Authors' contributions}

R-RZ carried out cell transplantation, immunoassays, ELISA and PCR analysis, participated in the drug metabolism and drafted the manuscript. Y-WZ participated in the design of the study and performed the statistical analysis. $\mathrm{BL}$ participated in the cell transplantation. TT performed the BrdU injection. 
YU carried out the microarray analysis. Y-ZN helped with raising mice and performed the liver biochemistry test. HT conceived of the study, and participated in its design and coordination and helped to draft the manuscript. All authors read and approved the final manuscript.

\section{Acknowledgements}

We thank the Mammalian Genetics Project, Tokyo Metropolitan Institute of Medical Science, for providing the mice. We also thank S Aoyama and $Y$ Adachi of ADME \& Toxicology Research Institute, Sekisui Medical Company Ltd, Japan, and K Kozakai and Y Yamada for assistance with LC-MS/MS analysis. This work was supported in part by Grants-in-Aid to Y-WZ (18591421, 20591531, and 23591872) from the Ministry of Education, Culture, Sports, Science, and Technology (MEXT), Japan; grants to Y-WZ for Strategic Research Projects (K18023 and K19023) of Yokohama City University, Japan; and grants to HT for Strategic Promotion of Innovative Research and Development (S-innovation, 62890004) from the Japan Science and Technology Agency (JST) and from the Center for Development of Innovative Technologies for metabolic organs using induced pluripotent stem cells (Type B) from the JST, Research Center Network for Realization of Regenerative Medicine. This research was supported in part by a research grant from the Ministry of Health, Labor, and Welfare of Japan.

\section{Author details}

'Department of Regenerative Medicine, Graduate School of Medicine, Yokohama City University, 3-9 Fuku-ura, Kanazawa-ku, Yokohama, Kanagawa 236-0004, Japan. ²Department of Advanced Gastroenterological Surgical Science and Technology, Faculty of Medicine, University of Tsukuba, Tsukuba 305-8575, Japan. ${ }^{3}$ Oregon Stem Cell Center, Oregon Health and Science University, Portland, OR 97239, USA. ${ }^{4}$ Advanced Medical Research Center, Yokohama City University, 3-9 Fuku-ura, Kanazawa-ku, Yokohama, Kanagawa 236-0004, Japan.

Received: 27 October 2014 Revised: 27 October 2014 Accepted: 5 March 2015 Published online: 26 March 2015

\section{References}

1. Muruganandan S, Sinal C. Mice as clinically relevant models for the study of cytochrome P450-dependent metabolism. Clin Pharmacol Ther. 2008;83:818-28.

2. Nishimura T, Hu Y, Wu M, Pham E, Suemizu H, Elazar M, et al. Using chimeric mice with humanized livers to predict human drug metabolism and a drug-drug interaction. J Pharmacol Exp Ther. 2013;344:388-96.

3. Suemizu H, Hasegawa M, Kawai K, Taniguchi K, Monnai M, Wakui M, et al. Establishment of a humanized model of liver using NOD/Shi-scid IL2Rgnull mice. Biochem Biophys Res Commun. 2008;377:248-52.

4. Tateno C, Yoshizane Y, Saito N, Kataoka M, Utoh R, Yamasaki C, et al. Near completely humanized liver in mice shows human-type metabolic responses to drugs. Am J Pathol. 2004;165:901-12.

5. Hasegawa M, Kawai K, Mitsui T, Taniguchi K, Monnai M, Wakui M, et al. The reconstituted 'humanized liver'in TK-NOG mice is mature and functional. Biochem Biophys Res Commun. 2011:405:405-10.

6. Azuma H, Paulk N, Ranade A, Dorrell C, Al-Dhalimy M, Ellis E, et al. Robust expansion of human hepatocytes in Fah-/-/Rag2-///I2rg-/- mice. Nat Biotechnol. 2007;25:903-10.

7. Rhim JA, Sandgren EP, Degen JL, Palmiter RD, Brinster RL. Replacement of diseased mouse liver by hepatic cell transplantation. Science. 1994;263:1149-52.

8. Haridass D, Yuan Q, Becker PD, Cantz T, Iken M, Rothe M, et al. Repopulation efficiencies of adult hepatocytes, fetal liver progenitor cells, and embryonic stem cell-derived hepatic cells in albumin-promoter-enhancer urokinase-type plasminogen activator mice. Am J Pathol. 2009;175:1483-92.

9. Sharma AD, Cantz T, Vogel A, Schambach A, Haridass D, Iken M, et al. Murine embryonic stem cell-derived hepatic progenitor cells engraft in recipient livers with limited capacity of liver tissue formation. Cell Transplant. 2008;17:313-23.

10. Peltz G. Can 'humanized'mice improve drug development in the 21st century? Trends Pharmacol Sci. 2013;34:255-60.

11. Zhu S, Rezvani M, Harbell J, Mattis AN, Wolfe AR, Benet LZ, et al. Mouse liver repopulation with hepatocytes generated from human fibroblasts. Nature. 2014;508:93-7.
12. Saito M, Iwawaki T, Taya C, Yonekawa H, Noda M, Inui Y, et al. Diphtheria toxin receptor-mediated conditional and targeted cell ablation in transgenic mice. Nat Biotechnol. 2001;19:746-50.

13. Ishii T, Yasuchika K, Machimoto T, Kamo N, Komori J, Konishi S, et al. Transplantation of embryonic stem cell-derived endodermal cells into mice with induced lethal liver damage. Stem Cells. 2007;25:3252-60.

14. Machimoto T, Yasuchika K, Komori J, Ishii T, Kamo N, Shimoda M, et al. Improvement of the survival rate by fetal liver cell transplantation in a mice lethal liver failure model. Transplantation. 2007;84:1233-9.

15. Taniguchi $\mathrm{H}$, Zheng YW. Human hepatic stem cell, method for preparation of the same, method for induction of differentiation of the same, and method for utilization of the same. http://www.google.com/patents/ US20110104126?hl=ja\&cl=en.

16. Mouse Genome Informatics. Gene Expression Database. http:// www.informatics.jax.org.

17. Takebe T, Sekine K, Enomura M, Koike H, Kimura M, Ogaeri T, et al. Vascularized and functional human liver from an iPSC-derived organ bud transplant. Nature. 2013;499:481-4.

18. Chen AA, Thomas DK, Ong LL, Schwartz RE, Golub TR, Bhatia SN. Humanized mice with ectopic artificial liver tissues. Proc Natl Acad Sci U S A. 2011;108:11842-7.

19. Si-Tayeb K, Noto FK, Nagaoka M, Li J, Battle MA, Duris C, et al. Highly efficient generation of human hepatocyte-like cells from induced pluripotent stem cells. Hepatology. 2010;51:297-305.

20. Ishizaki T, Sasaki T, Suganuma T, Horai Y, Chiba K, Watanabe M, et al. Pharmacokinetics of ketoprofen following single oral, intramuscular and rectal doses and after repeated oral administration. Eur J Clin Pharmacol. 1980;18:407-14.

21. Yu A-M, Idle JR, Gonzalez FJ. Polymorphic cytochrome P450 2D6: humanized mouse model and endogenous substrates. Drug Metab Rev. 2004;36:243-77.

22. Strom SC, Davila J, Grompe M. Chimeric mice with humanized liver: tools for the study of drug metabolism, excretion, and toxicity. Methods Mol Biol. 2010;640:491-509.

23. Bateman TJ, Reddy VG, Kakuni M, Morikawa Y, Kumar S. Application of chimeric mice with humanized liver for study of human-specific drug metabolism. Drug Metab Dispos. 2014;42:1055-65.

24. Zhang D, Luo G, Ding X, Lu C. Preclinical experimental models of drug metabolism and disposition in drug discovery and development. Acta Pharmaceutica Sinica B. 2012;2:549-61.

25. Nowak G, Ericzon B, Nava S, Jaksch M, Westgren M, Sumitran-Holgersson S. Identification of expandable human hepatic progenitors which differentiate into mature hepatic cells in vivo. Gut. 2005;54:972-9.

26. Liu Y, Yang R, He Z, Gao W-Q. Generation of functional organs from stem cells. Cell Regeneration. 2013;2:1.

\section{Submit your next manuscript to BioMed Central and take full advantage of:}

- Convenient online submission

- Thorough peer review

- No space constraints or color figure charges

- Immediate publication on acceptance

- Inclusion in PubMed, CAS, Scopus and Google Scholar

- Research which is freely available for redistribution 